\title{
Experiencia y manejo de dos casos de gangrena de Fournier en pacientes pediátricos en el Instituto Nacional de Salud del Niño - San Borja
} Experience and management of two cases of fournier's
gangrene in pediatric patients at the Instituto Nacional
de Salud del Niño - San Borja, Lima, Peru

Correspondencia Martín Humberto Iglesias Guzmán mig0013@hotmail.com

\section{Recibido: 09/06/2021}

Arbitrado por pares

Aprobado: 29/11/2021

Citar como: Iglesias-Guzmán $\mathrm{MH}$ Miranda del Carpio J, BustamanteLozada A, De Pawlikowski Amiel NW, Caller Farfán V, Medina Castillo JM. Experiencia y Manejo de dos casos de Gangrena de Fournier en pacientes pediatricos en el Instituto Nacional de Salud del Niño - San Borja. Acta Med Peru. 2021;38(4) 319-23. doi: https://doi.org/10.35663/ amp.2021.384.2134
Martín Humberto Iglesias Guzmán ${ }^{1, a}$, Juan Miranda del Carpio ${ }^{1, a}$, Atenas Bustamante Lozada ${ }^{1, a}$, Normy Wieslawa De Pawlikowski Amiel ${ }^{1, b}$, Verónica Caller Farfán ${ }^{1, b}$, Juan Manuel Medina Castillo ${ }^{1, b}$.

Instituto Nacional de Salud del Niño- San Borja, Lima. Perú.

Médico Residente de Cirugía Plástica.

Médico Cirujano Cirugía Plástica y Reconstructiva.

\section{RESUMEN}

La gangrena de Fournier es una patología que se encuentra predominantemente en varones adultos y extremadamente rara en niños. Se han descrito múltiples factores predisponentes en los niños, incluyendo la circuncisión, la dermatitis del pañal, la presencia de abscesos, traumatismos anorrectales y deficiencias inmunológicas. Los signos y síntomas característicos incluyen edema e hiperemia de rápida evolución en la región perineal acompañados de dolor intenso y fiebre. Una vez que se diagnostica la gangrena de Fournier, se debe instaurar tratamiento de forma inmediata, antibióticos endovenosos de amplio espectro y debridamiento quirúrgico temprano del tejido desvitalizado. A continuación presentamos un reporte de casos que incluye las características clínicas y epidemiológicas de dos pacientes pediátricos con gangrena de Fournier que recibieron tratamiento médico y quirúrgico en el Instituto Nacional de Salud del Niño de San Borja.

Palabras Clave: Gangrena de Fournier; Fascitis Necrotizante; Niños; Escroto; Perú. (Fuente: DeCS). 


\begin{abstract}
Fournier's gangrene is a condition mainly found in adults and it very rarely occurs in children. Multiple predisposing factors have been identified for children, including circumcision, diaper dermatitis, the occurrence of abscesses, anorectal trauma, and immune deficiency. Characteristic signs and symptoms include rapidly progressing edema and hyperemia in the perineal region, accompanied by intense pain and fever. Once Fournier's gangrene is diagnosed, therapy must be immediately instituted, using wide spectrum intravenous antibiotics and early surgical debridement of devitalized tissues. We present a case report including clinical and epidemiological characteristics of two pediatric patients with Fournier's gangrene who received medical and surgical therapy at the Instituto Nacional de Salud del Niño in San Borja, Lima, Peru.
\end{abstract}

Key words: Fournier's Gangrene; Necrotising Fasciitis; Children; Scrotum; Peru. (Source: MeSH).

\section{INTRODUCCIÓN}

La gangrena de Fournier (GF) es una fascitis necrotizante que afecta las regiones perianal y genital produciendo celulitis extensa con afectación grave del tejido subcutáneo, la fascia y el músculo o ambos, llevando a necrosis ${ }^{[1]}$. Afecta mayormente a adultos varones con un pico de incidencia entre 50 y 60 años, siendo una entidad infrecuente en la población pediátrica ${ }^{[2]}$. En los niños, los factores predisponentes incluyen traumatismos, picaduras de insectos, circuncisión, quemaduras, enfermedades periuretrales y anorrectales, infecciones sistémicas, inmunosupresión y neoplasias hematológicas ${ }^{[3]}$. La etiología bacteriana más frecuente es el sinergismo de anaerobios y aerobios, siendo estreptococo el organismo aislado con mayor frecuencia en pacientes pediátricos, siendo más frecuente en menores de 3 meses con una tasa de mortalidad de hasta el $7 \%{ }^{[4,5]}$.

El diagnóstico de la GF es principalmente clínico ${ }^{[6]}$. La alteración de algunos valores de exámenes de laboratorio, como la elevación de leucocitos, proteína $\mathrm{C}$ reactiva, creatinina y la disminución de albúmina y hematocrito; se han relacionado con un pronóstico desfavorable. Se describió el indicador de riesgo de laboratorio para fascitis necrotizante - LRINEC, una escala capaz de determinar casos tempranos de fascitis necrotizante ${ }^{[7,8]}$. El examen histológico revela necrosis de la fascia superficial y profunda, coagulación fibrinoide en la luz de los vasos, infiltración de tejidos polimórficos y detritos necróticos. El hallazgo patognomónico es la trombosis de los vasos que alimentan a los tejidos de una determinada localización ${ }^{[7]}$.

Una vez diagnosticada, el tratamiento debe iniciarse de inmediato: manejo del medio interno, antibioticoterapia endovenosa de amplio espectro y debridamiento quirúrgico del tejido desvitalizado hasta obtener tejido viable ${ }^{[2]}$. Pueden ser necesarias múltiples limpiezas quirúrgicas, resultando en pérdida significativa de tejido blando que requiere reconstrucción. Los objetivos de la reconstrucción de los defectos de GF son: proporcionar una cobertura protectora de los testículos, preservar la función testicular y obtener resultados cosméticos aceptables con una mínima morbilidad y mortalidad asociadas ${ }^{[9]}$. El presente estudio tiene como objetivo presentar las características clínicas, así como el manejo clínico y quirúrgico de dos casos de GF en pacientes pediátricos manejados en el Instituto Nacional de Salud del Niño de San Borja (INSN-SB).

\section{REPORTE DE CASOS}

Se describen las características clínicas, epidemiológicas, el manejo médico (Tabla I) y quirúrgico (Tabla II) de 2 casos de pacientes pediátricos con GF del INSN-SB.

\section{CASO 1}

Paciente varón de 30 días de vida presenta aumento de volumen escrotal izquierdo. Luego de punción de drenaje frustra, se presenta edema escrotal, pélvico y de miembro inferior izquierdo asociado a coloración violácea. Siete días después es referido al INSN-SB, presentando irritabilidad; los exámenes de laboratorio evidencian leucocitosis, aumento de proteína $C$ reactiva (PCR), hipoglucemia; con sospecha de GF (Score LRINEC: 6 puntos) (Tabla III). Ingresa a la Unidad de Cuidados Intensivos (UCI) e inicia tratamiento antibiótico con meropenem, vancomicina y metronidazol. Se realiza limpieza quirúrgica donde se evidencia: herida en escroto a predominio izquierdo de $7 \times 7 \mathrm{~cm}$ ulcerada, tejido necrótico en bordes que llega a músculos bulbocavernosos, secreción purulenta en volumen de $20 \mathrm{ml}$, abundante fibrina sobre cuerpos cavernosos y paredes internas del escroto. Se le realizaron 3 limpiezas quirúrgicas: 2 días, 4 días y 7 días después del ingreso, evolucionando favorablemente; por lo que pasa a hospitalización donde se realizan curaciones interdiarias, evidenciándose un defecto de piel escrotal de $45 \%$ (Figura ํ01). Al décimo catorce día del ingreso se realiza limpieza quirúrgica y cierre de defecto con colgajo de avance escrotal. A los 16 días del ingreso presenta neumonía aspirativa y pasa a la $\mathrm{UCl}$, iniciando antibioticoterapia con meropenem y vancomicina, donde permaneció 3 días. En hospitalización se realizan curaciones diarias evidenciándose zona operatoria afrontada, sin signos de flogosis, micción espontánea y 27 días después del ingreso es dado de alta. 
Tabla I. Características clínicas, epidemiológicas y manejo médico de dos casos de gangrena de Fournier en pacientes pediátricos del Instituto Nacional de Salud del Niño de San Borja.

\begin{tabular}{|c|c|c|}
\hline & CASO 1 & CASO 2 \\
\hline Edad & 30 días & 45 días \\
\hline Sexo & masculino & masculino \\
\hline Factor de riesgo & Aspiración con aguja & Dermatitis pañal \\
\hline Score LRINEC & 6 puntos & 8 puntos \\
\hline Anatomopatología (AP) & No se realizó & $\begin{array}{c}\text { Inflamación severa y trombosis de vasos pequeños } \\
\text { (endarteritis obliterante) }\end{array}$ \\
\hline Diagnóstico & Clínico & Clínico + AP \\
\hline Tratamiento antibiótico & $\begin{array}{l}\text { Meropenem+ Vancomicina + } \\
\text { Metronidazol }\end{array}$ & Meropenem+Vancomicina+ Clindamicina \\
\hline $\begin{array}{l}\text { Complicaciones durante } \\
\text { hospitalización }\end{array}$ & Neumonía aspirativa & Ninguno \\
\hline $\begin{array}{l}\text { Estancia en Unidad de Cuidados } \\
\text { Intensivos }\end{array}$ & 11 días & 6 días \\
\hline Estancia Hospitalaria total & 27 días & 25 días \\
\hline
\end{tabular}

Score LRINEC: Escala de Indicador de Riesgo Laboratorial para Fascitis Necrotizante

\section{CASO 2}

Paciente varón de 45 días de vida, presenta eritema en región perianal que progresa a aumento de volumen que se extiende a pene, escroto y a tercio distal de muslos. Un día después de iniciados los síntomas ingresa al INSN-SB en mal estado general, febril, taquicárdico; los exámenes de laboratorio evidencian leucocitosis con desviación izquierda, aumento de PCR, anemia moderada, aumento de tiempo de protrombina, con alta sospecha de GF (Score LRINEC: 8 puntos) (Tabla II). Se decide su traslado a $\mathrm{UCl}$ instaurándose antibioticoterapia con vancomicina, meropenem y clindamicina, apoyo inotrópico

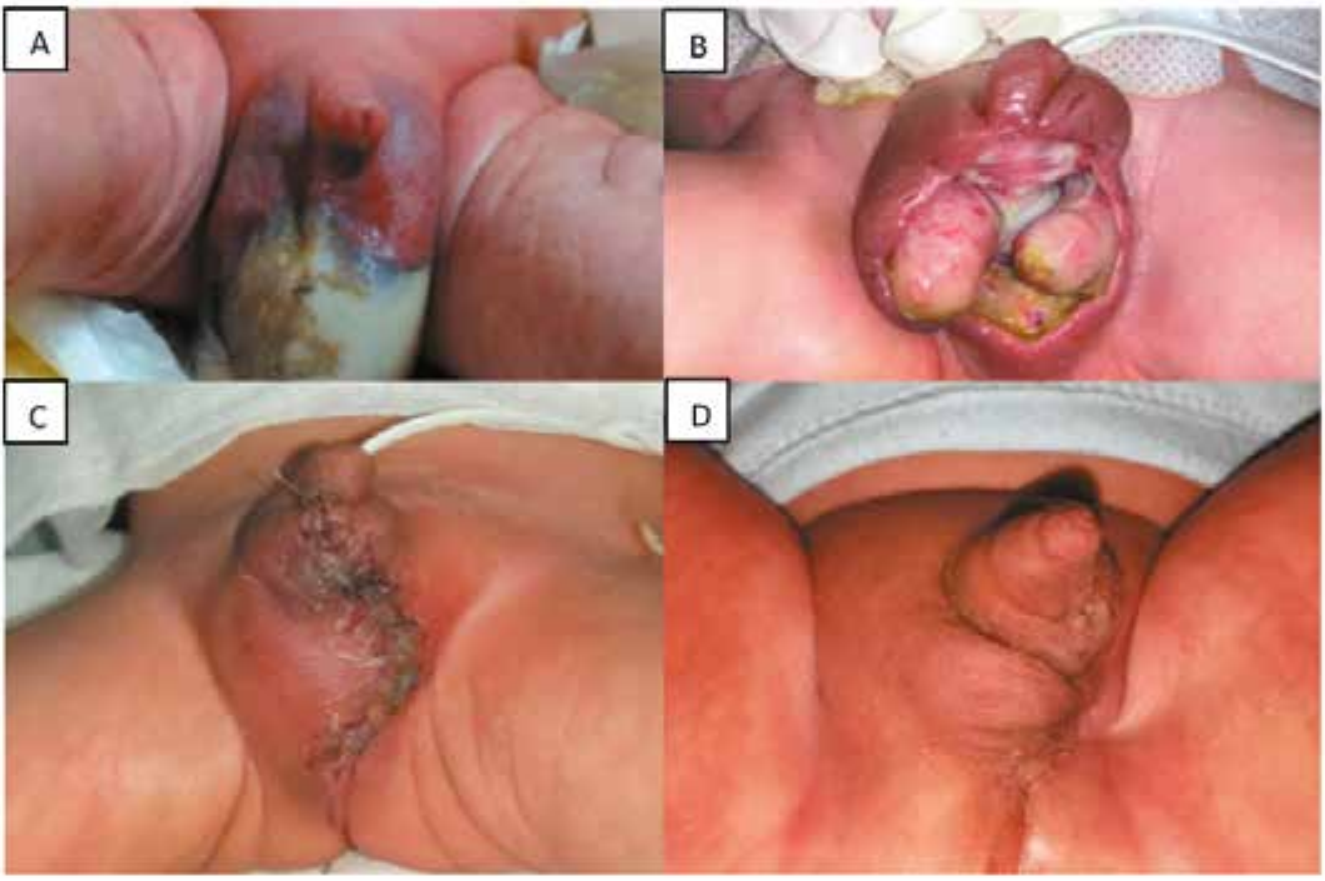

Figura $\mathbf{N}^{\circ}$ 01. Caso 1: varón de 30 días de vida. A. Al ingreso. B. Dos semanas después del ingreso, evidenciándose defecto escrotal de $45 \%$. C. 7 días luego de cierre de defecto con colgajo de avance escrotal. D. Un mes después de cierre de defecto. 
Tabla II. Manejo quirúrgico de dos casos de gangrena de Fournier en pacientes pediátricos del Instituto Nacional de Salud del Niño de San Borja.

\begin{tabular}{ccc} 
& CASO 1 & CASO 2 \\
\hline Tiempo de inicio de clínica a intervención quirúrgica & 7 días & 3 días \\
Número de intervenciones quirúrgicas & 5 & 3 \\
Número de limpiezas quirúrgicas & 5 & 3 \\
Porcentaje de defecto escrotal & $45 \%$ & $20 \%$ \\
Cirugía de reconstrucción & colgajo de avance escrotal & colgajo de avance escrotal \\
\hline
\end{tabular}

con noradrenalina, transfusión de plasma fresco congelado y fitomenadiona. Dos días después del ingreso, se realiza limpieza quirúrgica con hallazgos: edema a nivel escrotal y de prepucio, tejido necrótico central inferior, tejidos internos parcialmente desvitalizados y secreción seropurulenta intraescrotal de $15 \mathrm{ml}$. La patología evidencia inflamación severa y trombosis de vasos pequeños (endarteritis obliterante). A los 6 días del ingreso se realiza segunda limpieza quirúrgica, se retira apoyo inotrópico y se decide transferencia a hospitalización, donde se realizan curaciones interdiarias. Se evidencia pérdida de tejido escrotal de $20 \%$. Ver en material suplementario en OJS de AMP. Quince días después de su ingreso se realiza cierre de defecto mediante colgajo escrotal de avance, posteriormente continúa con curaciones diarias, y cursa con evolución favorable siendo dado de alta a los 25 días de ingreso.

\section{DISCUSIÓN}

La GF es una patología infrecuente en la población pediátrica, entre los factores predisponentes se encuentran la reacción alérgica al pañal, presencia de abscesos, traumatismos a nivel anorrectal y alteraciones inmunológicas ${ }^{[2,3]}$; incluso en el $40 \%$ de los casos son idiopáticos ${ }^{[10]}$. En nuestros pacientes, el primer caso presentado tuvo como antecedente punción en región perineal mientras que en el segundo paciente presentó dermatitis de pañal.

El diagnóstico de GF es principalmente clínico aunque los exámenes de laboratorio, imagenológicos e histopatológicos ayudan a confirmar el diagnóstico ${ }^{[6]}$. El indicador de riesgo de laboratorio para la fascitis necrotizante (LRINEC) es una puntuación de medición de laboratorio sólida capaz de determinar incluso los casos clínicamente tempranos de fascitis necrotizante. Una puntuación $\geq 6$ debe indicar sospecha de fascitis necrotizante y una puntuación de $\geq 8$ es un fuerte predictor de esta enfermedad ${ }^{[7,8]}$. Según el score de LRINEC, el primer caso tuvo una puntuación de 6 mientras que el segundo caso tuvo una puntuación de 8, correlacionando las manifestaciones clínicas y laboratoriales con GF. En la mayoría de casos, el cuadro clínico evoluciona de manera muy rápida y de no ser diagnosticado a tiempo conlleva rápidamente a la muerte ${ }^{[8]}$. Como vimos en el segundo caso presentado, la sintomatología inicia un día antes de su ingreso requiriendo manejo intensivo con apoyo inotrópico por cuadro de shock séptico aunque llegó a su resolución.

Tabla III. Escala de Indicador de Riesgo Laboratorial para Fascitis Necrotizante (score LRINEC) de dos casos de gangrena de Fournier en pacientes pediátricos del Instituto Nacional de Salud del Niño de San Borja.

\begin{tabular}{|c|c|c|c|c|}
\hline \multirow{2}{*}{ Variables (Unidades) } & \multicolumn{2}{|c|}{ CASO 1} & \multicolumn{2}{|c|}{ CASO 2} \\
\hline & Valor & Score & Valor & Score \\
\hline Proteína C reactiva (mg/L) & 180 & 4 & 352 & 4 \\
\hline Leucocitos $\left(\mathrm{mm}^{3}\right)$ & 15300 & 1 & 25060 & 2 \\
\hline Hemoglobina (g/dL) & 11.5 & 1 & 8.2 & 2 \\
\hline Sodio (mmol/L) & 137 & 0 & 138 & 0 \\
\hline Creatinina ( $\mu \mathrm{mol} / \mathrm{L})$ & 26.5 & 0 & 35.3 & 0 \\
\hline Glucosa (mmol/L) & 2.6 & 0 & 4.4 & 0 \\
\hline Total Score & & 6 puntos & & 8 puntos \\
\hline
\end{tabular}

La puntuación máxima es 13; una puntuación de 6 o más debe suscitar la sospecha de fascitis necrotizante y una puntuación de 8 es un fuerte predictor de esta enfermedad. 
El uso de antibióticos de amplio espectro a dosis adecuadas y el debridamiento quirúrgico temprano y agresivo del tejido necrótico son la base fundamental para controlar esta infección ${ }^{[8]}$. Como se vio en los casos presentados la mejoría clínica estuvo de la mano con el uso adecuado de antibioticoterapia y manejo quirúrgico de las lesiones, gracias a ello se pudo progresar de manera favorable hasta el cierre y reconstrucción de ambas lesiones. La reconstrucción escrotal es un reto; sin embargo, múltiples autores concuerdan que defectos de menos del $50 \%$ se pueden reconstruir realizando un cierre directo del defecto, 0 colgajos locales escrotales. Defectos mayores del $50 \%$ requieren reconstrucciones complejas que pueden incluir colgajos locales, regionales e inclusive colgajos libres ${ }^{[11]}$. Dado que la pérdida de tejido en nuestros dos pacientes fue de $45 \%$ y $20 \%$, realizamos un colgajo de avance escrotal en ambos casos con resultados favorables.

\section{CONCLUSIONES}

No hemos encontrado trabajos de investigación a nivel nacional que reporten casos de GF en pacientes pediátricos. Describimos el curso clínico, evolución y tratamiento de pacientes lactantes con $\mathrm{GF}$, haciendo énfasis en la necesidad de un tratamiento clínico quirúrgico temprano. Se espera que sirva como base para estudios futuros, considerando la rápida evolución y alta mortalidad de esta enfermedad. La información que se obtenga permitiría la creación de guías de manejo para esta patología en pacientes pediátricos.

Contribuciones de los autores: MHIG, JMDC, ABL, NWDPA. Diseño del manuscrito, recolección, análisis o interpretación de los datos. Redacción y revisión crítica importante del contenido del manuscrito. Aprobación final de la versión que se publicará. VCF y JMMC. Redacción y revisión crítica del manuscrito

Potenciales conflictos de Interés: Los autores no presentan conflicto de intereses para declarar.

\section{Fuente de financiamiento: Autofinanciado}

\section{ORCID}

Martín Humberto Iglesias Guzmán, https://orcid.org/0000-00022799-7483

Juan Miranda del Carpio, https://orcid.org/0000-0002-6209-3885

Atenas Bustamante Lozada, https://orcid.org/0000-0003-48360761

Normy Wieslawa De Pawlikowski Amiel, https://orcid.org/00000003-3331-1166

Verónica Caller Farfán, https://orcid.org/0000-0003-3707-4882 Juan Manuel Medina Castillo, https://orcid.org/0000-00024034-8648

\section{REFERENCIAS BIBLIOGRÁFICAS}

1. Villanueva-Sáenz E, Martínez Hernández-Magro P, Valdés Ovalle M, Montes Vega J, Alvarez-Tostado F JF. Experience in management of Fournier's gangrene. Tech Coloproctol. 2002;6(1):5-10; discussion 11-3.

2. Rouzrokh M, Tavassoli A, Mirshemirani A. Fournier's Gangrene in Children: Report on 7 Cases and Review of Literature. Iran J Pediatr. 2014;24(5):660-1.

3. Ekingen $G$, Isken T, Agir H, Oncel S, Günlemez A. Fournier's gangrene in childhood: a report of 3 infant patients. J Pediatr Surg. 2008;43(12):e39-42.

4. Adams JR Jr, Mata JA, Venable DD, Culkin DJ, Bocchini JA Jr. Fournier's gangrene in children. Urology. 1990;35(5):439-41.

5. Ameh EA, Dauda MM, Sabiu L, Mshelbwala PM, Mbibu HN, Nmadu PT. Fournier's gangrene in neonates and infants. Eur J Pediatr Surg. 2004;14(6):418-21.

6. Thwaini A, Khan A, Malik A, Cherian J, Barua J, Shergill I, et al. Fournierís gangrene and its emergency management. Postgraduate medical journal. 2006; 82:516. doi: 10.1136/pgmj.2005.042069

7. Chernyadyev SA, Ufimtseva MA, Vishnevskaya IF, Bochkarev YM, Ushakov AA, Beresneva TA, et al. Fournier's Gangrene: Literature Review and Clinical Cases. Urol Int. 2018;101(1):91-7.

8. Wong $\mathrm{CH}$, Khin LW, Heng KS, Tan KC, Low CO. The LRINEC (Laboratory Risk Indicator for Necrotizing Fasciitis) score: a tool for distinguishing necrotizing fasciitis from other soft tissue infections. Crit Care Med. 2004;32(7):1535-41.

9. Karian LS, Chung SY, Lee ES. Reconstruction of Defects After Fournier Gangrene: A Systematic Review. Eplasty. 2015;15:e18.

10. Gangopadhyay AN, Pandey A, Upadhyay VD, Sharma SP, Gupta DK Kumar V. Neonatal necrotising fasciitis--Varanasi experience. Int Wound J. 2008;5(1):108-12.

11. Khan Q, Knight R, Goodwin-Walters A. Scrotal reconstruction: a review and a proposed algorithm. Eur J Plast Surg. 2013;36:399-406. 